\title{
Studies on the Decrease Mechanisms of Typical Complex Networks
}

\author{
Yuhu QIU a, Tianyang LYU b, ${ }^{\text {, }}$ Xizhe ZHANG $^{\text {c, d }}$, Ruozhou WANG ${ }^{\text {d }}$ \\ ${ }^{a}$ University of International Relations, Beijing 100091, China \\ ${ }^{b}$ IT Center, National Audit Office, Beijing 100073, China \\ ${ }^{c}$ School of Biomedical Engineering and Informatics, Nanjing Medical University, \\ Nanjing 211166, China \\ ${ }^{d}$ School of Computer Science and Engineering, Northeastern University, \\ Shenyang110819, China
}

\begin{abstract}
Network decrease caused by the removal of nodes is an important evolution process that is paralleled with network growth. However, many complex network models usually lacked a sound decrease mechanism. Thus, they failed to capture how to cope with decreases in real life. The paper proposed decrease mechanisms for three typical types of networks, including the ER networks, the WS small-world networks and the BA scale-free networks. The proposed mechanisms maintained their key features in continuous and independent decrease processes, such as the random connections of ER networks, the long-range connections based on nearest-coupled network of WS networks and the tendency connections and the scale-free feature of BA networks. Experimental results showed that these mechanisms also maintained other topology characteristics including the degree distribution, clustering coefficient, average length of shortest-paths and diameter during decreases. Our studies also showed that it was quite difficult to find an efficient decrease mechanism for BA networks to withstand the continuous attacks at the high-degree nodes, because of the unequal status of nodes.
\end{abstract}

Keywords. Complex network, decrease mechanism, network evolution

\section{Introduction}

The running of complex systems like human society, biosphere rely on the interactions among large number of objects that form those systems. Complex network emerged as an important tool to study the interactions among objects since 1990s [1,2]. It has been widely applied in sociology [3], physics [4], biology [5], virology [6], information science [7] and etc.

Researchers proposed complex network models to simulate the interactions and evolutionary features of lots of objects/nodes. As one of the earliest network model, ER model supposed that nodes interacted with each other randomly [8]. Then, dynamic models with evolutionary features were proposed. Barabasi and Albert [9] proposed the scale-free models that embedded the preferential attachment rule for growth and simulated the rich-get-richer phenomenon in the degree distribution of complex networks.

\footnotetext{
${ }^{1}$ Corresponding author; Tianyang LYU, IT Center, National Audit Office, Beijing 100073, China; E-mail: raynor1979@163.com.
} 
These have become the foundations to understand, control and manipulate complex networks [6].

Network decreases referred to the removal, disappearance or deletion of nodes [10] that may lead to massive decrease of a network. In network evolution, decrease is a process that has the same importance as growth. A growth mechanism enabled a network expanded in suitable cases, while a decrease mechanism helped a network survive in harsh time [11]. Decreases have happened frequently in our normal life [11, 12], such as the decapitation strike on key individuals in a terrorist network, the financial crisis caused by the bankruptcy of Lehman Brothers, the failure of some nodes that lead to power grid breakdown in Europe and American. Previous studies [11-12] have figured out the influences of node removal and these influences varied with networks. Albert et al. [11] showed that random failures of nodes in random connection networks had accumulated consequences, but this was not the case for scale-free networks. Cohen et al. [12] showed that scale-free networks were vulnerable for attacks on high-degree nodes, and the weakness strengthened with the decrease of a network's power exponent. Hobbs and Burke [13] showed that attacks on specific nodes caused a cascade failure.

Complex networks in real life usually have a kind of coping strategy with network decreases, including replacement of a dying node, adjustments of interactions. For instance, terrorists always rebuilt their organizations after decapitation, and the social network also recovered from the death of an individual [10]. Therefore, it was critical for network models to contain a decrease mechanism. In the paper, a decrease mechanism referred to a strategy maintaining the existing network function with low cost when some nodes disappeared or even a network experienced a massive decrease.

However, many typical network models lacked a decrease mechanism. Take the scale-free model as an example. Although some experts proposed the decrease mechanism like preferential depletion [14], the mechanism was based on a fully connected network instead of a scale-free network. Bauke et al. [15] proposed to delete some nodes in network growth. In this case, decrease was still a by-product of growth, instead of an independent process that may lead to net reduction of network.

The paper proposed the corresponding independent decrease mechanisms for three typical models, including ER random network [8], WS small-world network [16] and BA scale-free network [9]. Each type of these networks has been widely found in the real life.

\section{Decrease Mechanisms of Typical Network Models}

A decrease mechanism should maintain topology features of a network. Topology features are usually critical for a network to perform its function and maintain its pattern. For instance, the existence of a few high-degree nodes greatly formed the propagation pattern of virus, information and action in a scale-free network, while the pattern was quite different in other types of networks [6]. Therefore, if a decrease made high-degree nodes vanish, the existed propagation mode would experience a big and costly change. From this perspective, the preferential attachment rule was sound that maintained the scale-free feature perfectly in growth. As a parallel mechanism, the decrease one should do the same job.

The key features of these typical networks that should be maintained in decrease were stated as follows. In general, the key feature of ER networks was random connections, this of WS networks was the long-range connection based on nearest- 
coupled networks, these of BA networks were the preferential attachment rule and the scale-free property. The paper firstly explored the decrease mechanisms maintaining these features. Since the number of edges $M$ of a certain number of nodes $N$ greatly determined other metrics, the paper supposed that the average degree $\bar{k}$ maintained before and after decrease of a network $G^{N}$.

As the removal of one node was the basis of network decreases, the paper proposed the decrease mechanisms of one node. It was quite simple to generalize the mechanisms to removal of lots of nodes.

\subsection{The Decrease Mechanism of ER Networks}

Nodes in an ER network $G_{E R}$ connected with each other under the same probability $P_{E R}^{N}=\frac{\bar{k}}{N-1}[8]$.

It was easy to prove that the removal of nodes in $G_{E R}$ led to over deletion of edges among remain nodes. Eq. (1) showed the number of edges $M^{\prime}$ after remove $\Delta \mathrm{N}$ nodes. But an ER network with the same $P_{E R}^{N}$ and $N-\Delta N$ nodes should have $M$ " edges as Eq. (2) showed. Therefore, the removal of $\Delta \mathrm{N}$ nodes results in over-deletion of $\Delta \mathrm{M}$ edges as Eq. (3) stated.

$$
\begin{gathered}
M^{\prime}=\frac{N \times \bar{k}}{2}-\left(\Delta N \times \bar{k}-\frac{\Delta N \times(\Delta N-1)}{2} \times P_{E R}^{N}\right) \\
M^{\prime \prime}=\frac{(N-\Delta N) \times \bar{k}}{2} \\
\Delta M=\frac{\bar{k} \times\left(N_{0}-\Delta N\right) \times \Delta N}{2 \times\left(N_{0}-1\right)} \\
\Delta M \stackrel{\Delta N=1 \bar{k}}{\longrightarrow}
\end{gathered}
$$

This reflected the disadvantage of survivors after a continuous decrease of an ER network. Edges of these remaining nodes became more and more sparse. And the degree distribution of survivors changed so greatly that the left network should no longer be considered as an ER network.

The proposed decrease mechanism of ER network was also simple. It randomly added $\bar{k} / 2$ edges among remaining nodes after remove one node as Eq. (4) shows. Then, the feature of ER network could maintain.

\subsection{The Decrease Mechanism of WS Networks}

A WS small-world network $G_{w s}$ was constructed based on a nearest-coupled network by replacing an edge with the probability $p_{w s}[16]$. During the replacement, an edge $e(i, j)$ replaced one of its end point $v_{i}$ and with another node. Since new end point had a high probability to be a node far from the remaining one, $G_{w s}$ showed small-world feature with short network diameter. And the replaced edge was usually called a long connection. 
The higher the $p_{w s}$, the smaller the diameter. According to the feature of nearest-coupled network, $\bar{k}$ was usually even.

There were two obvious influences of the removal of a node $v_{i}$ in a WS network. First, the removal of $v_{i}$ broke the nearest-couple relationships of $v_{i}$ 's neighbors. Second, if $v_{i}$ was the end point of some long-connections, vanish of many $v_{i}$ s would influence the small-world feature of $G_{w s}$. Figure $1(a)$ and $1(b)$ showed an example, removal of $v_{0}$ influenced the neighbor relation between $v_{0}$ and $v_{6}$ and the long-connection $e(4,0)$. To be more clearly, the paper placed nodes in a circle and the nearest neighbors of a node were placed at its both sides as Figure 1 showed.

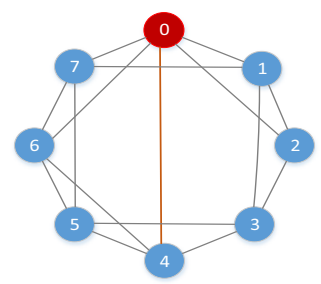

(a)

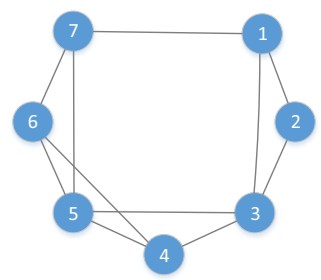

(b)

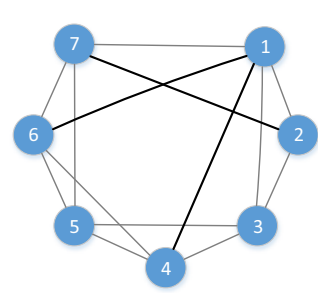

(c)

Figure 1. A WS network with $\bar{k}=4$ changed before and after the removal of $v_{0}$. (a) Original network (b) The network after $v_{0}$ was deleted. (c)The network processed by the proposed decreased mechanism.

Therefore, the follow-up process was needed. Firstly, some edges of $v_{i}$ should be replaced by other edges to maintain coupled relations of $v_{i}$ 's nearest neighbors. Easy to prove that $\bar{k} / 2$ edges should be deleted and $\bar{k} / 2$ edges should be replaced. In the paper, if $\bar{k} / 2$ was even, $\bar{k} / 4$ edges at both sides of $v_{i}$ were deleted and replaced respectively. If $\bar{k} / 2$ was odd, $(\bar{k}+2) / 4$ edges were removed and $(\bar{k}-2) / 4$ edges were replaced at the left side of $v_{i}$, and $(\bar{k}-2) / 4$ edges were removed and $(\bar{k}+2) / 4$ edges were replaced at the right side.

Secondly, handle the long-connections related with $v_{i}$. The strategy mainly focused on whether the original edge corresponding to a long-connection was planned to be deleted or replaced after decrease. The long-connection should be deleted if the original edge was, or the long-connection should be replaced.

Thus, we proposed the decrease mechanism of WS networks after the removal $v_{i}$ as follows.

(1) Edge deletion.

- In the case of $\bar{k} / 2$ is even, delete all edges $e(i, j)$, if $j \in\{i-\bar{k} / 4, \ldots, i+\bar{k} / 4\}$ and $i \neq j$;

- In the case of $\bar{k} / 2$ is odd, delete all edges $e(i, j)$, if $j \in\left\{i-\frac{\bar{k}+2}{4}, \ldots, i+\frac{\bar{k}-2}{4}\right\}$ and $i \neq j$;

(2) Edge replacement.

- In the case of $\bar{k} / 2$ is even, replace all edges $e(i, j)$, if $j$ satisfies the following requirements and $i \neq j$ and $e(i, j)$ is not replaced by a long-connection before decrease; when $j \in\left\{i-\frac{\bar{k}}{2}, i-\frac{\bar{k}}{2}+1 \ldots i-\frac{\bar{k}}{4}-1\right\}$, replace $e(i, j)$ by $e\left(j+\frac{\bar{k}}{\underline{2}}+\right.$ $1, j)$; when $j \in\left\{i+\frac{\bar{k}}{4}+1, i+\frac{\bar{k}}{4}+2 \ldots i+\frac{\bar{k}}{2}\right\}$, replace $e(i, j)$ by $e\left(j-\frac{\bar{k}}{2}-\right.$ $1, j)$; 
- In the case of $\bar{k} / 2$ is odd, replace all edges $e(i, j)$, if $j$ satisfies the following requirements and $i \neq j$ and $e(i, j)$ is not replaced by a long-connection before decrease; when $j \in\{i-\bar{k} / 2, i-\bar{k} / 2+1, i-(\bar{k}+2) / 4-1\}$, replace $e(i, j)$ by $e\left(j+\frac{\bar{k}}{2}+1, j\right)$; when $j \in\{i+(\bar{k}-2) / 4+1, i+(\bar{k}-2) / 4+2 \ldots, i+$ $\bar{k} / 2\}$, replace $e(i, j)$ by $e\left(j-\frac{\bar{k}}{2}-1, j\right)$;

(3) Handle long-connections.

- When the edge $e(i, j)$ to be replaced in the above process is already replaced by a long-connection $e(i, p)$ or $v_{i}$ becomes an end point of a long-connection, replace $e(i, p)$ by $e(i-1, p)$ or $e(i+1, p)$ randomly; when $e(i, j)$ is replaced by a long-connection $e(j, p)$, removal of $v_{i}$ has no influence on the longconnection and no more action needed.

- When $v_{i}$ was a faraway node of $e(i, p)$ after replaced the original edge $e(q, p)$, replace $e(i, p)$ by $e(i-1, p)$ or $e(i+1, p)$ randomly.

- Randomly delete $\Delta N \times \frac{\bar{k}}{2} \times p_{w s}$ long-connections to maintain $p_{w s}$ during decrease, where $\Delta N$ is the number of removal nodes.

Figure 1(c) showed an example. With $\bar{k}=4, e(0,7)$ and $e(0,1)$ were deleted; $e(0,6)$ was replaced by $e(1,6)$ and $e(0,2)$ was replaced by $e(7,2)$; the long-connection $e(0,4)$ was replaced by $e(1,4)$.

Using this mechanism, a WS network could maintain its nearest-coupled and small world features. But the decrease mechanism was much more complicated than that of ER. It had to perform complex edge replacements around a deleted node and keep tracking the status of at most $N \times \frac{\bar{k}}{2} \times p_{w s}$ long-connections.

\subsection{The Decrease Mechanism of BA Networks}

The major feature of BA networks was rooted in the preferential attachment in network growth, which means new node tended to connect with high-degree nodes. This made a network evolved the scale-free property. Thus, the earlier a node joined a network, the higher its degree might be.

Therefore, the key of a decrease mechanism was to maintain the existence of highdegree nodes, especially the early nodes with high-degree. Easy to prove that, for a BA network $G_{B A}$ with $\bar{k}$, the degree of a new node was $\bar{k} / 2$. If the degree of a node $v_{j}$ larger than $\bar{k} / 2$, it was caused by connections added by nodes latter than $v_{j}$. Based on this analysis, the basic idea of the proposed mechanism was to delete edges connected at the moment when $v_{j}$ joined the network, but rebuilt those connected after $v_{j}$. Thus, it looked like $v_{j}$ has never existed when newcomers after $v_{j}$ joined the network.

Therefore, the decrease mechanism of BA network was stated as follows,

(1) In the case of the degree of $v_{j} k_{j}=\bar{k} / 2$, delete $v_{j}$ and its edges.

(2) In the case of $k_{j}>\bar{k} / 2, k_{j}-\bar{k} / 2$ edges should be rebuilt. Let the set of nodes joined before $v_{j}$ record as $A_{j-}$ and the set of $v_{j}$ 's neighbors that belong to $A_{j-}$ record as $B_{j-}$; let the set of nodes joined before $v_{j}$ record as $A_{j+}$ and the set of $v_{j}$ 's neighbors that belong to $A_{j+}$ record as $B_{j+}$. According to the growth mechanism of a BA network, $\left|B_{j-}\right|=\bar{k} / 2$ and $\left|B_{j+}\right|=k_{j}-\bar{k} / 2$. The rebuilding rule is:

(a) Randomly select a node $v_{i}$ from $B_{j+}$; 
(b) Select a high-degree node $v_{k}$ from $A_{j-}$ according to the preferential attachment rule and no edge existing between $v_{k}$ and $v_{i}$, rebuild $e(k, i)$;

(c) Delete $v_{i}$ from $B_{j+}$;

(d) Repeat (a)-(c) until $B_{j+}$ is empty.

Figure 2 showed an example where nodes were numbered according to their sequence joining the network and $v_{0}$ was the first. When a newcomer $v_{7}$ was deleted, only $e(7,1)$ and $e(7,4)$ were deleted. But when $v_{4}$ was deleted, the edges created by adding $v_{5}$, $v_{6}, v_{7}$ should be rebuilt. Thus, $e(6,4)$ was replaced by $e(6,0), e(5,4)$ was replaced by $e(5,2)$ and $e(8,4)$ was replaced by $e(8,2)$.

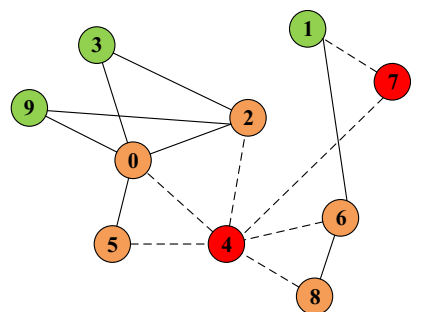

(a)

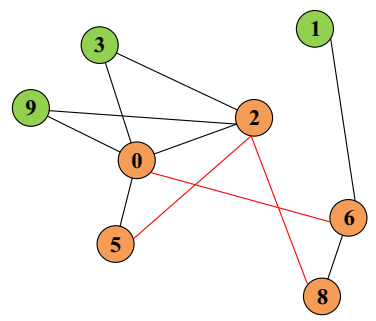

(b)

Figure 2. A BA network with $\overline{\mathrm{k}}=4$ changes before and after the removal of $v_{4}$ and $v_{7}$ (a) Original network

(b) The rebuilt network after the removal of $v_{4}$ and $v_{7}$.

This decrease mechanism maintained the local connectivity around a removal node and the high-degree property of first comers in a BA network. In comparison with those of ER and WS networks that have no growth mechanism like BA networks, the BA mechanism needed the time-dependent global information of the joining sequence of all nodes, while the mechanism of ER needed no additional information and that of ER needed only global information of a few long-connections. However, such global information was often rare for the Internet and social networks. Moreover, the performance of this mechanism to cope with continuous attacks on high-degree nodes still needed further verification.

\section{Experiments and Analysis}

In experiments, we verified whether the proposed mechanisms maintained the topology characteristics like degree distribution, clustering coefficient, average length of shortestpaths and diameter during decrease, as they maintained the major network features.

For each type of networks, we first generated 5 initial networks with $N_{0}=10000$; all these networks decreased until $N=1000$. To avoid the influence of randomness, the decrease process was performed 20 times independently and the average of these metrics of the decreased networks were used in comparison with those of new created networks with the same size. If their metrics were similar, it means decreases caused no significant influence on a network. Let $1000 \_$n correspond to a new network with $N=1000$, while $1000 \_$m corresponded to a network decreased from initial to $N=1000$. For BA networks, we also analyzed their performances when there were continuous attacks on high-degree nodes. And 1000_d corresponded to a BA network decreased after target attacks. 


\subsection{Decrease Experiments of ER and WS Networks}

The proposed mechanisms of ER and WS networks performed well in the experiments. Figure 3, Figure 4 and Figure 5 showed that the metrics of new ER or WS networks were quite similar with those of the decreased networks, in the cases when the sizes decreased to 7000,4000 and even 1000 . The results also showed that the proposed mechanisms maintained the key features of ER and WS networks. Only WS networks showed a little difference in metrics including the clustering coefficient and the average length of shortest-paths when networks decreased to the small size in Figure 5. It may be caused by the influence of decreases on long-connections.

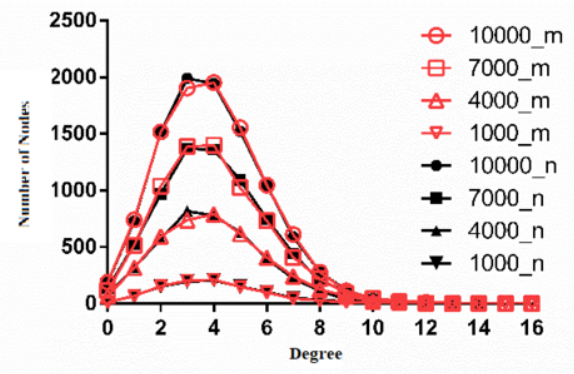

(a)

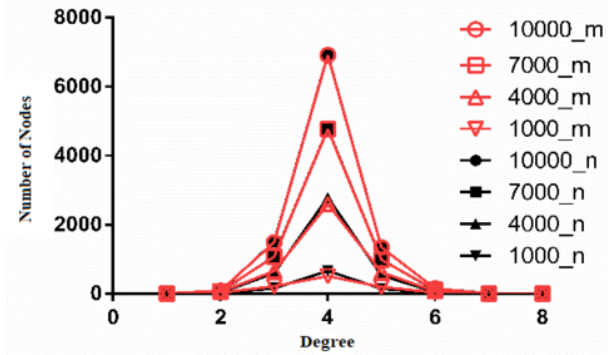

(b)

Figure 3. Degree distribution comparisons of ER and WS networks before and after decrease. (a) Degree distribution comparisons of ER networks. (b) Degree distribution comparisons of WS networks.
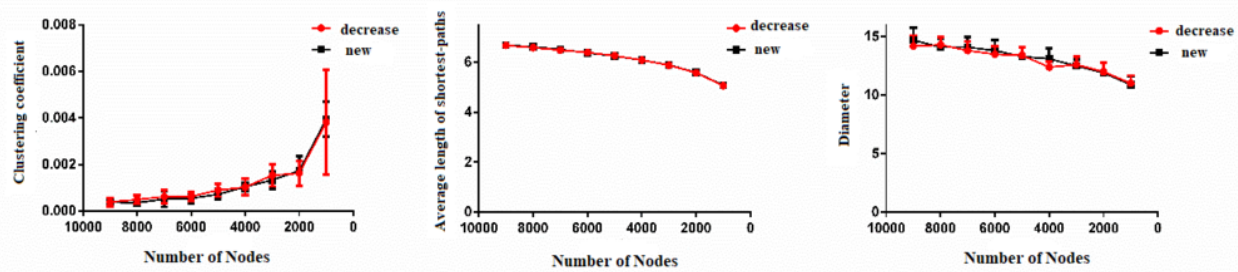

Figure 4. Topology metrics comparisons of ER networks with and without decreases.
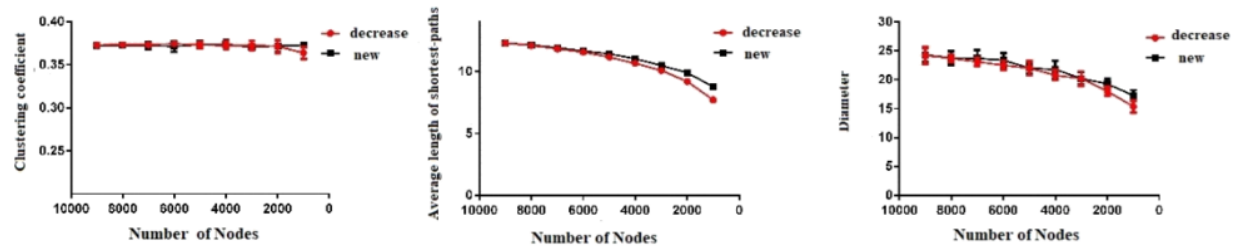

Figure 5. Topology metrics comparisons of WS networks with and without decreases. 


\subsection{Decrease Experiments of BA Networks}

Since there were clear differences of node status in a BA network, we analyzed the decrease performances after random deletion, and after target deletion of high-degree nodes. The results showed that the proposed mechanism handled the random deletion quite well. The metrics of new BA networks were quite similar with the networks decreased to 9000, 5000 and 1000 in Figure 6 and Figure 7.

However, due to BA's intrinsic drawback, the mechanism was not good enough to encounter the continuous attacks on high-degree nodes. Figure 6 showed that the long tail vanished after the removal of 1000, 5000 and 9000 high-degree nodes. Figure 8 further showed the serious consequences on other metrics after continuous target attacks, where minus_ondegree means target attacks and standard means new created BA networks. Preliminary analysis showed that the degree of target deleted nodes $k_{j}>>\bar{k} / 2$. In this case, lots of edges were rebuilt, but the preferential attachment process cannot guarantee the existence of too many super star nodes. This led to a relative fair distribution of degree. Therefore, the reconstruction of all edges of a decreased network could solve this problem. However, the cost was too heavy to be applicable in real life.
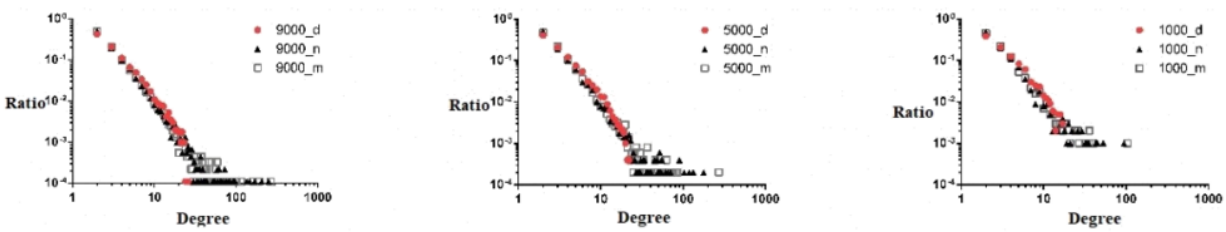

Figure 6. Changes of the degree distribution after random and target deletion of nodes of BA networks.
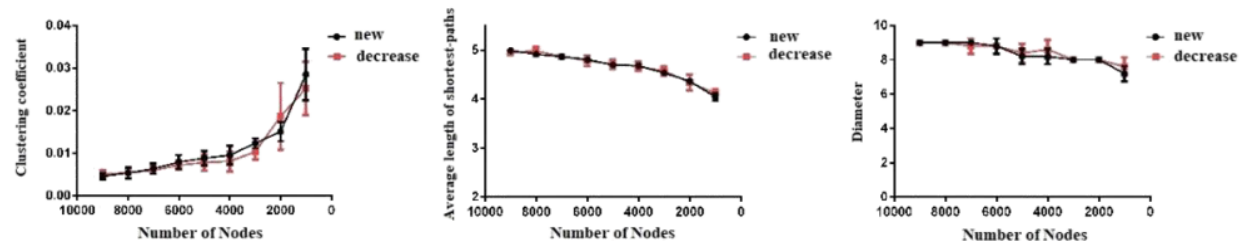

Figure 7. Changes of the degree distribution after random deletion of nodes of BA networks.
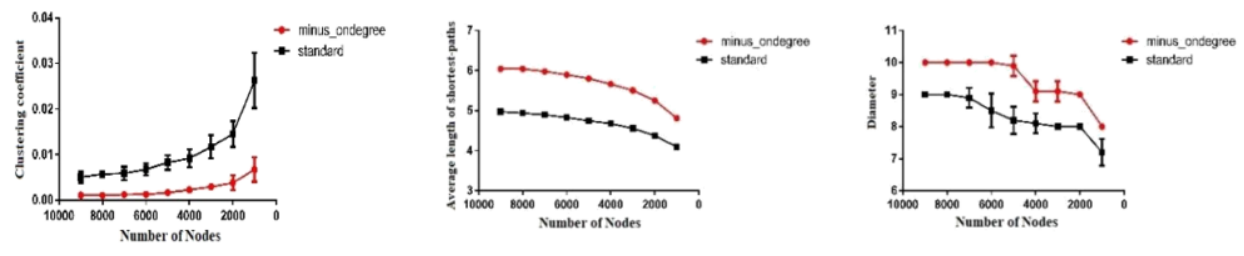

Figure 8. Changes of topology properties after target deletion of nodes of BA networks. 


\section{Conclusion}

The paper proposed the decrease mechanisms for three typical complex networks including ER, WS and BA. The study showed their good performance in maintaining the key features and topology characteristics of all these networks in decrease. However, we also found that some mechanisms also need local or even global information to handle the complex cases emerged after nodes' removal. From this perspective, decrease may be much more complex than growth that needs more attentions. Moreover, a sound decrease one for BA networks that can withstand continuous target attacks was still open for discussion, and detail observations of real networks on their decrease processes were critical for future studies.

\section{Acknowledgement}

This work was partially supported by the Natural Science Foundation of China under grant number 91546110 and by the Scientific Research Funds for the Construction of High-grade, Precision and Advanced Discipline of University of International Relations under grant number 2019GA32.

\section{References}

[1] Barabási A, Crandall RE. Linked: the new science of networks. Amer. J. Phys. 2002; 71: 243-270.

[2] Watts DJ. The "new" science of networks. Annual Rev. Sociol. 2004; 30:243.

[3] Latora V, Marchiori M. Efficient behavior of small-world networks. Phys. Rev. Lett. 2001; 87:198701.

[4] Albert R, Barabási A. Statistical mechanics of complex networks. Rev. Modern Phys. 2001; 74: xii.

[5] Daura-Jorge FG, Cantor M, Ingram SN, Lusseau D, Simões-Lopes PC. The structure of a bottlenose dolphin society is coupled to a unique foraging cooperation with artisanal fishermen. Biol. Lett. 2012; 8: 702.

[6] Pastorsatorras R, Vespignan A. Epidemic spreading in scale-free networks. Phys. Rev. Lett. 2001; 863200.

[7] Maslov S, Sneppen K, Zaliznyak A. Pattern detection in complex networks: correlation profile of the internet. Chromosoma. 2002; 2002(3): 113-115.

[8] Erdős P, Rényi A. On random graphs I. Publicationes Mathematicae 1959; 6: 290-297.

[9] Barabási AL, Albert R. Emergence of scaling in random networks. Science 1999; 286: 509.

[10] Motter AE, Lai YC. Topology of the conceptual network of language. Phys. Rev. E Statistical Nonlinear Soft Matter Physics. 2002; 66: 065102.

[11] Albert R, Jeong H, Barabasi AL. Error and attack tolerance of complex networks. Nature, 2001; 340: 388-394.

[12] Cohen R, Erez K, Ben-Avraham D. Breakdown of the internet under intentional attack. Phys. Rev. Lett. 2001; 86: 3682 .

[13] Hobbs WR, Burke MK. Connective recovery in social networks after the death of a friend. Nature Human Behaviour. 2017; 1: 0092.

[14] Schneider CM, Arcangelis LD, Herrmann HJ. Scale free networks by preferential depletion. EPL 2011; 95: 1305-1323.

[15] Bauke H, Moore C, Rouquier JB, Sherrington D. Topological phase transition in a network model with preferential attachment and node removal. The European Phys. J. B. 2011; 83(2011): 519.

[16] Watts DJ, Strogatz SH. Collective dynamics of 'small-world' networks. Nature. 1998; 393: 440. 